\title{
Test bench technologies for improving WLTP measurement results
}

Zdenek Mestenhauser, V. Senft

MTS Systems Corporation

This manuscript is not available according to publishing restriction.

Thank you for your understanding.

(C) Springer Fachmedien Wiesbaden 2016, M. Bargende, H.-C. Reuss, J. Wiedemann (Hrsg.), 16. Internationales Stuttgarter Symposium, Proceedings, DOI 10.1007/978-3-658-13255-2_2 